\title{
Sol-Gel Transition of Sodium Gellan Gums with Different Molar Masses in Aqueous Solutions
}

\author{
E. Ogawa ${ }^{1}$, K. Yamazaki $^{2}$, K.Sugimoto ${ }^{2}$, \\ R.Takahashi ${ }^{3}$, K.Kubota ${ }^{3}$, H.Yajima ${ }^{4}$, and K. Nishinari ${ }^{5}$ \\ ${ }^{1}$ Showagakuin Jr. College, Higashisugano, Ichikawa, Chiba 272-0823, Japan. \\ Fax: 81-47-322-6800, e-mail: QYK04426@nifty.ne.jp, \\ ${ }^{2}$ Eko Instruments Co.,Ltd. Technical Center, Shibuya, Tokyo, 151-0072 \\ Fax: 81-3-3469-5897, e-mail: sugimoto@eko.co.jp \\ ${ }^{3}$ Graduate School of Engineering, Gunma University, Kiryu, Gunma 376-8515, Japan \\ Fax:81-277-30-1442, e-mail: rheo@bce.gunma-u.ac.jp \\ ${ }^{4}$ Faculty of Science, Tokyo University of Science, Kagurazaka, Shinjyuku-ku, Tokyo,162-8601, Japan. \\ Fax: 81-3-3235-2214, e-mail: yajima@ rs.kagu.tus.ac.jp \\ ${ }^{5}$ Graduate School of Human Life Science, Osaka City University, Sugimoto, Sumiyoshiku, Osaka 558-8585, Japan. \\ Fax:81-6-6605-3086, e-mail: nisinari@life.osaka-cu.ac.jp
}

Using three kinds of well-purified sodium type gellan gums (Na-gellan, $M_{\mathrm{w}}=12.7 \times 10^{3}, 9.7 \times 10^{3}, 7.1 \times 10^{3}$ ), we studied the effects of molar mass on the sol to gel transition and the coil to double-helix transition in aqueous solutions with and without $\mathrm{NaCl}, \mathrm{KCl}$, and $\mathrm{CaCl}_{2}$ by rheological measurements. In aqueous solutions with and without salts, the temperature dependence of storage modulus $\left(G^{\prime}\right)$ and loss modulus $\left(G^{\prime \prime}\right)$ for the three sample solutions of various polymer concentrations $\left(c_{\mathrm{p}}=0.5-5.0 \%\right)$ was measured from 60 to 5 ${ }^{\circ} \mathrm{C}$. In aqueous solutions without salts $\left(c_{\mathrm{p}}=0.5-5.0 \%\right)$, the coil to double-helix transition temperature $\left(T_{\mathrm{ch}}\right)$ for the three Na-gellans were the same, however, the sol to gel transition temperature $\left(T_{\mathrm{sg}}\right)$ depended markedly on molar mass; $T_{\mathrm{sg}}$ of Na-gellan samples decreased with decreasing molar mass. In aqueous solutions with salts $\left(c_{\mathrm{p}}=1.0 \%\right), T_{\mathrm{sg}}$ and $T_{\mathrm{ch}}$ overlapped, indicating that the sol to gel transition and the coil to double-helix transition for Na-gellans (GF1-GF3) occurred concurrently. It was found that, in salt solutions, $T_{\mathrm{ch}}$ and $T_{\mathrm{sg}}$ were almost independent of molar mass.

Key words: gellan gum, sol-gel transition, coil-helix transition, aqueous solution, rheology

\section{INTRODUCTION}

Gellan gum, an anionic polysaccharide, undergoes gelation by forming domains composed of associated double-helices [1]. Previously, using well-purified sodium-type gellan gums (Na-gellans) with different molar masses, we studied the coil to helix conformational transition in aqueous solutions with and without $25 \mathrm{mM}$ $\mathrm{NaCl}$ by, light scattering (LS), viscosity, and circular dichroism (CD) measurements and differential scanning calorimetry (DSC)[2,3]. Using three kinds of wellpurified Na-gellans with different molar masses, the effects of molar mass on the sol to gel transition for
Na-gellans in aqueous solutions without salt were studied by $\mathrm{CD}$, viscosity, and rheological measurements [4].

In this study, using well-purified three Na-gellan samples which were used in our previous study [4], we investigated the effects of molar mass and salts such as $\mathrm{NaCl}, \mathrm{KCl}$, and $\mathrm{CaCl}_{2}$ on the sol to gel transition and the coil to helix transition for Na-gellan in aqueous solutions by rheological measurements.

\section{EXPERIMENTAL}

Materials Na-gellan samples (GF1-GF3) were prepared from the deacetylated gellan gum (San-Ei Gen 
F.F.I., Osaka, Japan) by sonication [4]. The molar mass of the samples are shown in Table 1. The characteristics of the samples were reported previously [4].

Measurements Rheological measurements were performed using an RS 600 (Thermo HAAKE) with parallel plate geometry of $60 \mathrm{~mm}$ diameter [4]. Temperature dependence of storage modulus, $G^{\prime}$, and loss modulus, $G^{\prime \prime}$, at a frequency of $0.3 \mathrm{~Hz}$ was observed by cooling the systems from 60 to $5{ }^{\circ} \mathrm{C}$ at a rate of 0.5 ${ }^{\circ} \mathrm{C} / \mathrm{min}$.

Table I Characteristics of the Na-gellan samples [4]

\begin{tabular}{cc}
\hline Sample & $M_{\mathrm{w}} \times 10^{-3}$ a) \\
\hline GF1 & 125 \\
GF2 & 95.3 \\
GF3 & 73.8 \\
\hline
\end{tabular}

a) Values are determined by LS measurements at $40^{\circ} \mathrm{C}$ in aqueous solutions with $25 \mathrm{mM} \mathrm{NaCl}$.

\section{RESULTS AND DISCUSSION}

In aqueous solutions without salt, temperature dependence of $G^{\prime}$ and $G^{\prime \prime}$ for the three sample solutions of various polymer concentrations $\left(c_{\mathrm{p}}=0.5-5.0 \%\right)$ was measured from 60 to $5{ }^{\circ} \mathrm{C}$. In addition, in order to know the rheological character, we made torque vs angle Lissajous plots (data not shown).
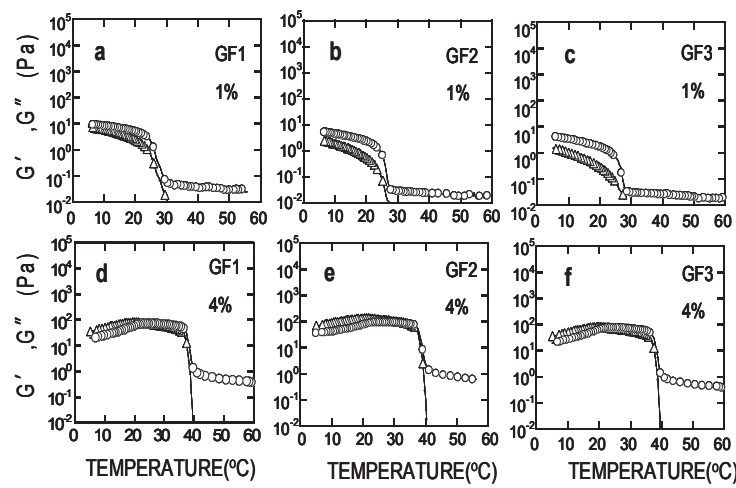

Fig.1 Temperature dependence of the storage modulus, $G^{\prime}$, and the loss modulus, $G^{\prime \prime}$, during the cooling process for the Na-gellan (GF1, GF2, and GF3) aqueous solutions without salts. $(\triangle) G^{\prime},(\bigcirc) G^{\prime \prime}$. (a)GF1,1\%; (b)GF2,1\%; (c)GF3, 1\%; (d)GF1,4\%; (e)GF2,4\%; (f)GF3,4\%.

Typical examples for the temperature dependence of $G^{\prime}$ and $G^{\prime \prime}$ are shown in Fig.1. In the cooling process, the temperature dependence of $G^{\prime \prime}$ for GF1-GF3 solutions showed a drastic increase at a certain temperature, and

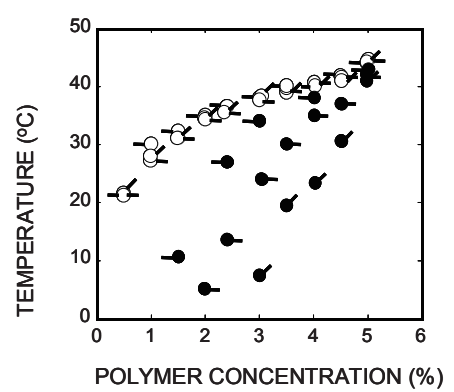

Fig.2 Polymer concentration dependence of $T_{\mathrm{ch}}$ and $T_{\mathrm{sg}}$ for Na-gellan (GF1, GF2, and GF3) aqueous solutions without salts. $\quad T_{\text {ch }}(-\bigcirc) \mathrm{GF} 1 ;(\bigcirc-) \mathrm{GF} 2 ;(\sigma)$ GF3; $T_{\mathrm{sg}}(\bullet) \mathrm{GF} 1 ;(\bullet-) \mathrm{GF} 2 ;(\bullet) \mathrm{GF} 3$.

$G^{\prime}$ was too small to be detected above that temperature. The steep increase in $G^{\prime \prime}$ in the thermal scanning rheology was attributed to the coil to double-helix transition, $T_{\mathrm{ch}}$, of Na-gellan molecules, and the crossover temperature of $G^{\prime}$ and $G^{\prime \prime}$ was attributed to the sol-gel transition $T_{\mathrm{sg}}[4,5]$. The values of $T_{\mathrm{ch}}$ and $T_{\mathrm{sg}}$ determined from temperature dependence of $G^{\prime}$ and $G^{\prime \prime}$ for GF1-GF3 are plotted against the polymer concentration in Fig.2. As shown in Fig 2, $T_{\text {ch }}$ for GF1, GF2, and GF3 are almost the same, suggesting that the temperature at which the coil to double-helix transition takes place does not depend on molar mass. Previously [2, 3] using five Na-gellan samples $\left(M_{\mathrm{w}}=32 \times 10^{3}-125 \times 10^{3}\right)$, we investigated the polymer concentration dependence of $T_{\mathrm{ch}}$ from $\mathrm{CD}$, viscometric, and rheological measurements and DSC in a concentration range $(<5 \%)$. It was concluded that values of $T_{\text {ch }}$ for the Na-gellan solutions were independent of molar mass in the molar mass range examined. In the present study, we obtained the same conclusion. On the other hand $T_{\mathrm{sg}}$ corresponding to the sol-gel transition of Na-gellan aqueous solution depends markedly on molar mass. Below $c_{\mathrm{p}}=5 \%, T_{\mathrm{sg}}$ decreased with decreasing molar mass; GF1 $>$ GF2 $>$ GF3 . At low concentrations of Na-gellan (below $c_{\mathrm{p}}=1.5 \%, 2 \%$, and $3 \%$ for GF1, GF2, and GF3, respectively) $T_{\mathrm{sg}}$ could not be observed. The coil to double-helix transition and the sol-gel transition occurred at different temperatures below $c_{\mathrm{p}}=5 \%$; the double-helices formation and its partial 
aggregation may form a certain ordered structure but does not lead to gel formation because the number of helical aggregates is not sufficient to form a three dimensional network. Above $c_{\mathrm{p}}=5 \%$ solutions, $T_{\mathrm{ch}}$ for the GF1, GF2, and GF3 overlap with $T_{\mathrm{sg}}$, indicating that the doublehelix formation and gel formation occurred concurrently. Dependence of $T_{\mathrm{sg}}$ on molar mass suggests that the gel formation of Na-gellan aqueous solutions is influenced strongly by the length of double-helices together with the number of double-helices. As shown in Fig. 1, for $c_{\mathrm{p}}=4 \%$ solutions, maxima of $G^{\prime}$ and $G^{\prime \prime}$ were observed above $T_{\mathrm{sg}}$, which depended on the cooling rates (data are not shown). Based on the observation of Lissajous figures, it was confirmed that slippage between sample and measuring geometry did not occur. It is considered that these maxima could be attributed to change in the microstructure of the gel network.

In order to investigate the effects of salts such as $\mathrm{NaCl}, \mathrm{KCl}$, and $\mathrm{CaCl}_{2}$ on the gelation of Na-gellans, temperature dependence of $G^{\prime}$ and $G^{\prime \prime}$ of the GF1-GF3 was measured in aqueous solutions $\left(c_{\mathrm{p}}=1 \%\right)$ with salts. Typical examples for the temperature dependence of $G^{\prime}$ and $G^{\prime \prime}$ are shown in Figs.3, 5, 7. The concentration of $\mathrm{NaCl}$ or $\mathrm{KCl}$ in solutions varied from 5 to $75 \mathrm{mM}$ and that of $\mathrm{CaCl}_{2}$ ranged from 1 to $2 \mathrm{mM}$. $T_{\mathrm{ch}}$ and $T_{\mathrm{sg}}$ are plotted against salts concentration in Figs. 4 and 6. In aqueous solutions with $\mathrm{NaCl}, \mathrm{KCl}$, and $\mathrm{CaCl}_{2}, T_{\mathrm{ch}}$ for GF1, GF2, and GF3 are almost the same, suggesting that $T_{\mathrm{ch}}$ does not depend on molar mass. Similar results were observed in aqueous solutions without salts as described above. However, $T_{\text {ch }}$ of the three samples in aqueous salts solutions are much higher than those in aqueous solutions without salts. In $1 \%$ solutions without salts, the crossover of $G^{\prime}$ and $G^{\prime \prime}$ for the GF1-GF3 was not observed within the temperature range from 60 to $5{ }^{\circ} \mathrm{C}$. While, 1\% Na-gellan (GF1-GF3) solutions with $\mathrm{NaCl}$ and $\mathrm{KCl}$ the sol-gel transition occurred except for the
GF2 and GF3 solutions of $50 \mathrm{mMNaCl}$ or $25 \mathrm{mMKCl}$. As shown in Figs.4 and 5, $T_{\mathrm{sg}}$ of the GF1 solutions with $25 \mathrm{mM} \mathrm{NaCl}$ or $5 \mathrm{mM} \mathrm{KCl}$ were around $5^{\circ} \mathrm{C}$.
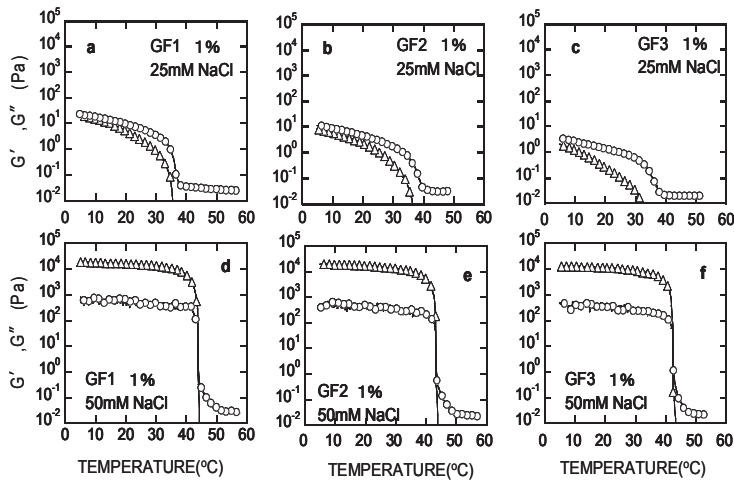

Fig.3 Temperature dependence of the storage modulus, $G^{\prime}$, and the loss modulus, $G^{\prime \prime}$, during the cooling process for the 1\% Na-gellan (GF1, GF2, and GF3) aqueous solutions with $\mathrm{NaCl}$. $(\triangle) G^{\prime}(\bigcirc) G^{\prime \prime}$, (a)GF1,25mM; (b)GF2,25mM; (c)GF3,25mM;

(e)GF2,50mM; (f) $\mathrm{GF} 3,50 \mathrm{mM}$.

(d)GF1,50mM;

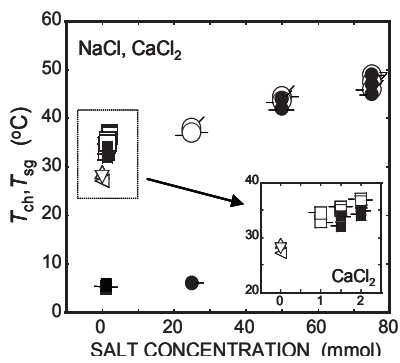

Fig.4 Salt concentration dependence of $T_{\mathrm{ch}}$ and $T_{\mathrm{sg}}$ for Na-gellan (GF1,GF2, and GF3) aqueous solutions with

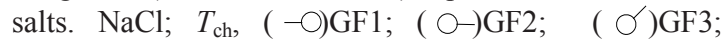
$T_{\mathrm{sg}},(-) \mathrm{GF} 1 ; \quad\left(\bullet-\mathrm{GF} 2 ; \quad(\bullet) \mathrm{GF} 3 ; \quad \mathrm{CaCl}_{2} ; T_{\mathrm{ch}}\right.$,

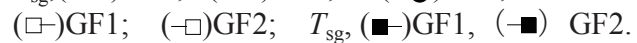

$\mathrm{H}_{2} \mathrm{O} ; \quad T_{\text {ch }},(\triangleleft) \mathrm{GF} 1 ; \quad(\triangle) \mathrm{GF} 2,(\nabla) \mathrm{GF} 3$.
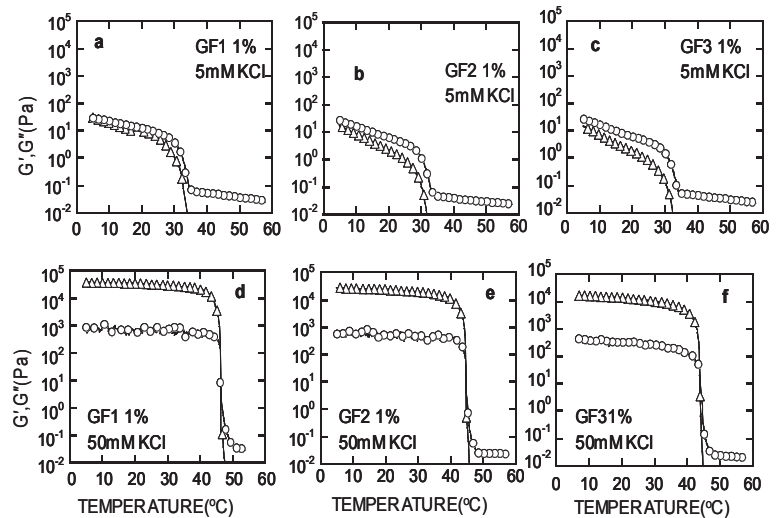

Fig. 5 Temperature dependence of the storage modulus, $G^{\prime}$, and the loss modulus, $G^{\prime \prime}$, during the cooling process for the $1 \%$ Na-gellan (GF1, GF2, and GF3) aqueous solutions with $\mathrm{KCl}$. $(\triangle) G^{\prime}(\bigcirc) G^{\prime \prime}$,
(a) GF1,5mM;
(b) GF2,5mM;
(c) $\mathrm{GF} 3,5 \mathrm{mM}$;

(d)GF1,50mM;

(e) GF2,50mM;

(f) $\mathrm{GF} 3,50 \mathrm{mM}$ 


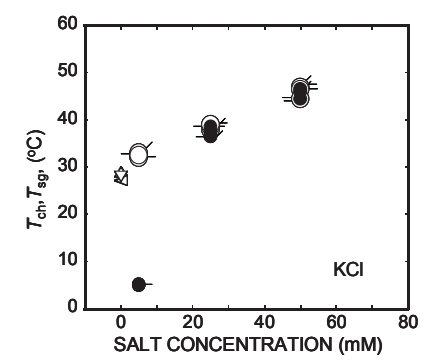

Fig.6 Salt concentration dependence of $T_{\mathrm{ch}}$ and $T_{\mathrm{sg}}$ for Na-gellan (GF1, GF2, and GF3) aqueous solutions with $\mathrm{KCl}$. $T_{\mathrm{ch}},(-\bigcirc) \mathrm{GF} 1 ;(\bigcirc-) \mathrm{GF} 2,\left(\bigcirc^{\prime}\right) \mathrm{GF} 3 ; T_{\mathrm{sg}},(-\bullet) \mathrm{GF} 1$, $(\bullet) \mathrm{GF} 2,(\bullet) \mathrm{GF} 3 . \mathrm{H}_{2} \mathrm{O} ; T_{\text {ch }},(\triangleleft) \mathrm{GF} 1,(\triangle) \mathrm{GF} 2,(\nabla)$

At high salt concentrations (above $c_{\mathrm{s}}=50 \mathrm{mM}$, and $25 \mathrm{mM}$ of $\mathrm{NaCl}$ and $\mathrm{KCl}$, respectively), crossover of $G^{\prime}$ and $G^{\prime \prime}$ attributed to the sol-gel transition temperature $T_{\mathrm{sg}}$ was observed, and $T_{\mathrm{sg}}$ and $T_{\mathrm{ch}}$ overlaps, indicating that the sol-gel transition and the coil-double-helix transition for Na-gellans (GF1-GF3) occurred concurrently. It was found that, in monovalent cation solutions such as 50 and $75 \mathrm{mMNaCl}$ and 25 and $50 \mathrm{mMKCl}, T_{\text {sg }}$ and $T_{\text {ch }}$ were independent of molar mass. $T_{\mathrm{sg}}$ and $T_{\mathrm{ch}}$ of the solutions with monovalent cations were higher than those of the solution without cations and increased with increasing monovalent cation concentrations. Moreover, by the addition of salts to $1 \%$ Na-gellan solutions without salts, the values of $G^{\prime}$ and $G^{\prime \prime}$ observed below the sol-gel transition temperature region increased significantly. It is found that the rheological behavior of Na-gellan solutions was influenced strongly by monovalent cations.

Comparison the results obtained in $\mathrm{NaCl}$ and $\mathrm{KCl}$ solutions shows that the $T_{\mathrm{sg}}\left(T_{\mathrm{ch}}\right)$ in $\mathrm{KCl}$ solutions were slightly higher than $T_{\mathrm{sg}}\left(T_{\mathrm{ch}}\right)$ in $\mathrm{NaCl}$ solutions with the same salt concentration. Below the sol-gel transition temperature, $G^{\prime}$ and $G^{\prime \prime}$ for the Na-gellan solutions with $50 \mathrm{mM} \mathrm{KCl}$ were larger than those of the solutions with $50 \mathrm{mM} \mathrm{NaCl}$. It is noted that the effect of $\mathrm{KCl}$ on viscoelasticity of Na-gellan solutions is stronger than that of $\mathrm{NaCl}$.

On addition of a slight amount $(1 \mathrm{mM})$ of $\mathrm{CaCl}_{2}$, the sol-gel transition for GF1 and GF2 solution occurred at 5 and $6{ }^{\circ} \mathrm{C}$, respectively. In the Na-gellan solutions with $1.5 \mathrm{mM}$ and $2 \mathrm{mM} \mathrm{CaCl}_{2}, T_{\text {sg }}$ overlapped with $T_{\text {ch }}$ and $T_{\text {sg }}$ which were independent of molar mass. While, in the monovalent cation solutions of low $c_{\mathrm{s}}(5 \mathrm{mMKCl}$ and $25 \mathrm{mMNaCl}$ ), the crossover of $G^{\prime}$ and $G^{\prime \prime}$ was not observed for GF2 and GF3, and the crossover of $G^{\prime}$ and
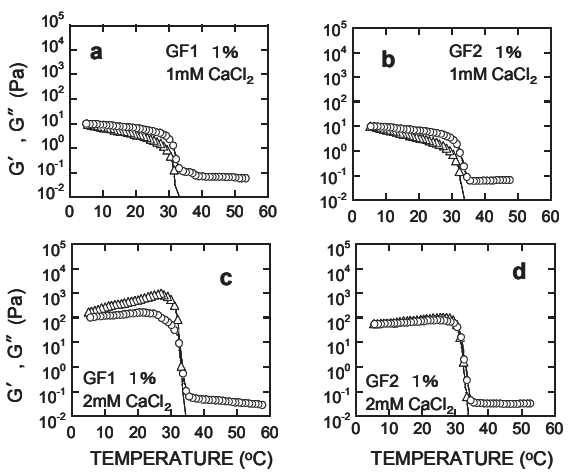

Fig.7 Temperature dependence of the storage modulus, $G^{\prime}$, and the loss modulus, $G^{\prime \prime}$, during the cooling process for the $1 \%$ Na-gellan (GF1, GF2, and GF3) aqueous solutions with $\mathrm{CaCl}_{2}$. $\quad(\triangle) G^{\prime} ; \quad(\bigcirc) G^{\prime \prime}$; (a)GF1 $1 \mathrm{mM}$; (b)GF2 1mM; (c)GF1 2mM; (d)GF2 2mM.

$G^{\prime \prime}$ was observed for GF1-GF3 solutions with high $c_{\mathrm{s}}$ $(50 \mathrm{mMNaCl}$ and $25 \mathrm{mM} \mathrm{KCl})$. From these results, it is suggested that the rheological behavior of Na-gellan solutions was effected more strongly by divalent cation of $\mathrm{Ca}^{2+}$ than by monovalent cations such as $\mathrm{Na}^{+}$and $\mathrm{K}^{+}$, as shown in a previous study [5]. It was found that $T_{\mathrm{sg}}$ for $1 \%$ Na-gellan solutions containing low $c_{\mathrm{s}}$ of divalent cations was independent on molar mass. Similar as the results obtained for $1 \%$ solutions with high $c_{\mathrm{s}}$ of monovalent cations. On the other hand, $T_{\mathrm{sg}}$ for Na-gellan solutions below $5 \%$ without cations depended markedly on molar mass.

\section{References}

1.K.te.Nijenhuis, "Thermoreversible Networks", Springer, Berlin, (1997) Chapter 13.

2. E.Ogawa, R.Takahashi, H.Yajima, K.Nishinari Biopolymers 79, 207-217(2005)

3. E.Ogawa, R.Takahashi, H.Yajima, K.Nishinari Food Hydrocolloids 20, 378-385(2006).

4. E.Ogawa, K.Sugimoto, R.Takahashi, H.Yajima, K. Kubota, K.Nishinari Trans. Mater. Res. Soc. Jpn. 31, 731-734 (2006).

5. E.Nishinari, T.Takaya, K.Nishinari Carbohydr. Polym. 30, 109-119(1996)

(Received April 28, 2009; Accepted June 9, 2009) 